\title{
SLINGSHOT PROMINENCES DURING DWARF NOVA OUTBURSTS?
}

\author{
D. STEEGHS ${ }^{1}$, K. HORNE ${ }^{2}$, T. R. MARSH ${ }^{3}$ \\ 1. Astronomical Institute, Utrecht University, The Netherlands \\ 2. Physics $\mathcal{E} 3$ Astronomy, University of St.Andrews, UK \\ 3. Astronomy Group, University of Southampton, UK
}

\begin{abstract}
We present phase-resolved spectroscopy of two dwarf novae, IP Peg and SS Cyg, observed in outburst with the WHT/ISIS spectropolarimeter. Doppler tomograms of several emission lines show peculiar narrow, stationary emission components.
\end{abstract}

\section{Introduction}

IP Peg is an eclipsing dwarf nova with an orbital period of $3.8 \mathrm{~h}$, and an outburst cycle of about three months. IP Peg was observed during outburst on 1993 August $27\left(0.37 \AA\right.$ pixel $\left.^{-1}\right)$. Secondary star emission was very strong during the outburst, and a peculiar emission component, with a very low velocity, was observed in the $\mathrm{H} \alpha$ line, visible as a spot in the Doppler map (Fig. 1, top left). SS Cyg is a U Gem-type dwarf nova with an orbital period of $6.6 \mathrm{~h}$, and an outburst cycle of about $50 \mathrm{~d}$. SS Cyg was observed for about $6 \mathrm{~h}$ on 1993 August 28 during an anomalous (slow) outburst, just after maximum light. The wavelength ranges $6390 \AA$ to $6850 \AA(0.37 \AA$ pixel $\left.^{-1}\right)$ and $4200 \AA$ to $5000 \AA\left(0.78 \AA\right.$ pixel $\left.^{-1}\right)$ were covered. Again lowvelocity emission components were observed in $\mathrm{H} \alpha$, He I (6678) and $\mathrm{He}$ II (4686) (see Fig. 1). Secondary star emission is strong in most lines, except in the He II (4686) line which is only visible during outburst, but which shows a very clear stationary emission component.

\section{Stationary emission components}

Both dwarf novae observed show low velocity components in their emission lines. The low velocities make it incompatible with secondary star emission or emission from the white dwarf although secondary star emission is observed to be strong during outburst.

Although there is no obvious part of the binary system moving with the observed velocity, a possible interpretation may be 'slingshot prominences'; 

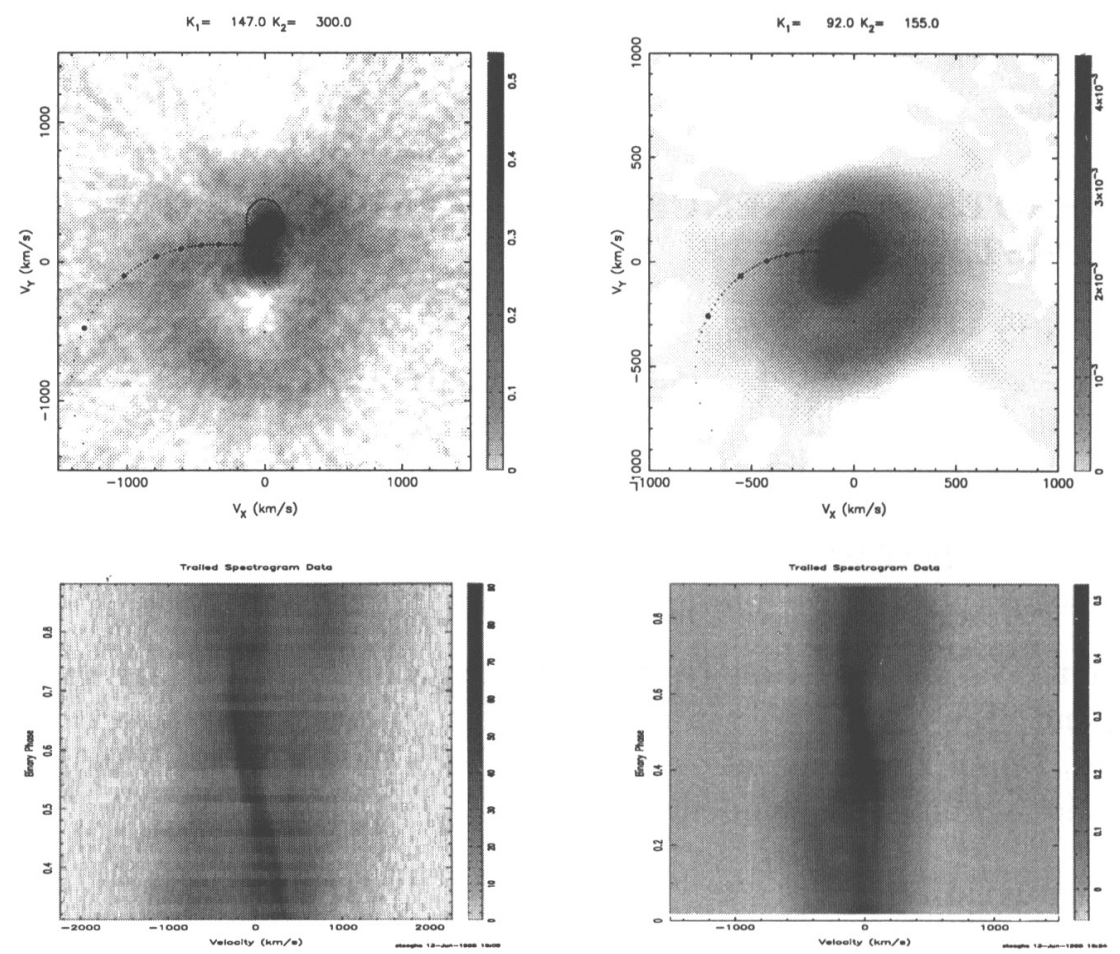

Figure 1. Top panels: Doppler tomograms constructed using the filtered back-projection method (Horne 1991). Left shows the $\mathrm{H} \alpha$ map for IP Peg, right $\mathrm{H} \alpha$ emission from SS Cyg. Apart from a ring of disk emission and emission from the secondary star, both systems display low velocity emission, outside the secondary star's Roche lobe. Bottom panels: Observed data in the form of trailed spectrograms.

material trapped in magnetic loops and co-rotating with the secondary star (similar to the prominences observed from single, rotating dwarf stars). In IP Peg the supposed prominence extends about a stellar radius beyond the $\mathrm{L}_{1}$ point into the Roche lobe of the white dwarf. Here gravity pulls material toward the top of the loop, where it can collect.

At this point we do not know whether our interpretation is correct, or how widespread such emission features may be during dwarf nova outbursts. Are large prominences always present, but just lit up during the outburst, or could they somehow be a cause or effect of the outburst? Work is in progress to constrain possible prominence parameters from the observed fluxes. In the case of IP Peg, eclipse observations can also be used to constrain the emission geometry.

\section{References}

Horne, K., 1991, in "Fundamental Properties of CVs" ed. A.W. Shafter (U. San Diego) 